\title{
External radiation treatment of malignant liver disease: a critical review
}

\author{
Angel I. Blanco • Bin S. Teh • Laura A. Dawson • \\ Ramiro Pino • Arnold C. Paulino • E. Brian Butler
}

Received: 24 February 2012 / Accepted: 16 May 2012 /Published online: 2 June 2012

(C) Springer-Verlag 2012

\begin{abstract}
Introduction Owing to the liver's integral role in biosynthesis and homeostasis, management of primary and secondary malignancies arising in this organ is of paramount oncologic significance. Over the past several decades, substantial progress has been achieved in the imaging and treatment of early and advanced hepatic malignancies. Radiation therapy (RT) has recently emerged as one of many local therapeutic options for both primary and metastatic liver cancer. Recent phase I and II studies describe promising efficacy and side effect profile; however, phase III studies are needed to establish RT among standard of care therapeutic modalities.

Purpose In this review, we will describe (a) the scope, epidemiology, and standard treatment options for liver cancer; (b) relevant diagnostic imaging techniques; and (c) provide an in-depth review of RT treatment techniques, dose-volume limits of normal tissues, and results of both conventional and hypofractionated RT liver trials pertaining to primary and secondary liver cancers with emphasis on three-dimensional conformal radiation therapy (3DCRT) and stereotactic body radiotherapy (SBRT) studies.
\end{abstract}

A. I. Blanco • B. S. Teh • R. Pino - A. C. Paulino - E. B. Butler Department of Radiation Oncology, The Methodist Hospital/The Methodist Hospital Research Institute,

Houston, TX, USA

\section{A. Dawson}

Radiation Medicine Program, Princess Margaret Hospital, Dept. of Radiation Oncology, University of Toronto,

Toronto, ON, Canada

\section{A. I. Blanco $(\bowtie)$}

Department of Radiation Oncology, The Methodist Hospital, 6565 Fannin, DB1-077,

Houston, TX 77030, USA

e-mail: blanco.angel@gmail.com
Keywords External radiation treatment $\cdot$ Malignant liver disease $\cdot$ Diagnostic imaging techniques $\cdot$ 3DCRT $\cdot$ SBRT

\section{Scope and management of hepatic malignancy}

Primary liver cancer

With an estimated incidence of 24,120 new cases in 2010, primary cancers of the liver and intrahepatic bile duct are relatively uncommon in the United States, accounting for approximately $3 \%$ of overall cancer mortality [1]. Worldwide, however, primary liver cancer (PLC) is endemic, with the highest incidence rates reported in East Asia and Central and West Africa [2]. During the last two decades, increasing PLC incidence has been reported in Australia, Central Europe, the UK, and North America. Leading risk factors for primary liver cancer include hepatitis B and C, alcohol, and tobacco. Diagnosis is either performed using multiphasic contrast-enhanced imaging, which is sufficient for diagnosis for hepatocellular carcinoma (HCC) patients with underlying liver disease if the classic imaging findings of early arterial phase enhancement and venous or delayed washout are found or, alternatively, biopsy [3]. Tumor markers including $\alpha$-fetoprotein (for HCC) or CA19-9 (for biliary carcinomas) are complimentary to imaging and may be used in the follow-up of patients.

Treatment options for HCC, the primary focus of this section, are stage-dependent, reflecting burden of disease and invasion of adjacent or distant structures. To date, the Child-Pugh system [4] remains one of the most frequently utilized classifications to describe liver function, which is important in treatment decision making and prognosis. Many staging systems include some measure of liver function (e.g., Okuda), although a description of their relative 
merits lies beyond the scope of this work. A common theme is that prognosis of primary liver tumors is dependent on both disease extent and underlying liver function.

As demonstrated in Fig. 1, summarizing the Barcelona Clinic classification (BLCL) [3], local therapies are considered potentially curative for patients in good performance status with "very early" (i.e., single tumors less than $2 \mathrm{~cm}$ ) or other early-stage tumors. Patients with very early-stage tumors may be amenable to surgical resection alone [5] whereas, for other patients with early disease (i.e., a single tumor $\leq 5 \mathrm{~cm}$ in diameter or $\leq 3$ tumors $\leq 3 \mathrm{~cm}$ in diameter), transplantation represents the optimal therapeutic approach. For such patients, the original Milan study [6] demonstrated overall and recurrence-free survival rates at 4 years of $85 \%$ and $92 \%$, respectively. This modality also synchronously addresses the underlying cirrhotic liver as well as the HCC. Alternatively, potentially curative treatment options also exist for patients with compensated cirrhosis or those unable to undergo transplantation. These include (a) upfront hepatic resection with delayed orthotopic liver transplant (OLT) (with published overall and disease-free survival rates of $69 \%$ and $44 \%$ at 5 years [7]); and (b) radiofrequency ablation (RFA), with 5-year survival rates of approximately $27 \%$, and with outcomes dependent on Child-Pugh class, tumor size, and achievement of an initial complete response [8]. RFA also benefits cirrhotic patients awaiting OLT as a form of "bridge" therapy.

Patients outside the Milan criteria, who unfortunately comprise more than $70 \%$ of $\mathrm{HCC}$ patients, are usually considered unresectable and incurable by current treatment modalities. These patients are typically divided into intermediate and advanced stages (BCLC groups B and C, Fig. 1). Standard options include transarterial chemoembolization (TACE) and systemic therapy. Individual TACE trials describe a survival benefit, in patients without major vascular involvement. A systematic review of randomized trials for unresectable HCC was subsequently conducted by Llovet et al. [9]. Among 328 studies, only 14 were suitable for analysis. Seven trials investigated TACE, and the remaining investigated the role of tamoxifen. TACE significantly improved 2 -year survival ( $41 \%$ vs $27 \%$ ) with objective responses in $35 \%$ of treated patients. Sensitivity analysis showed a significant benefit of chemoembolization with cisplatin or doxorubicin but none with embolization alone. In contrast, tamoxifen showed no statistically significant benefit in 1-year survival.

Two phase III trials established sorafenib as an effective systemic treatment for unresectable or metastatic disease. The first (SHARP) trial [10] randomized 602 patients to receive sorafenib $400 \mathrm{mg}$ twice daily versus placebo. Patients assigned to sorafenib had improved disease control rates $(43 \%$ vs. $32 \%)$, median time to radiographic progression (5.5 vs 2.8 months), and overall survival (10.7 vs 7.9 months). These results were confirmed in the second phase III trial [11], in which sorafenib improved median survival from 4.2 to 6.5 months in HCC patients from Asia, predominantly with hepatitis B. Treatment-related side

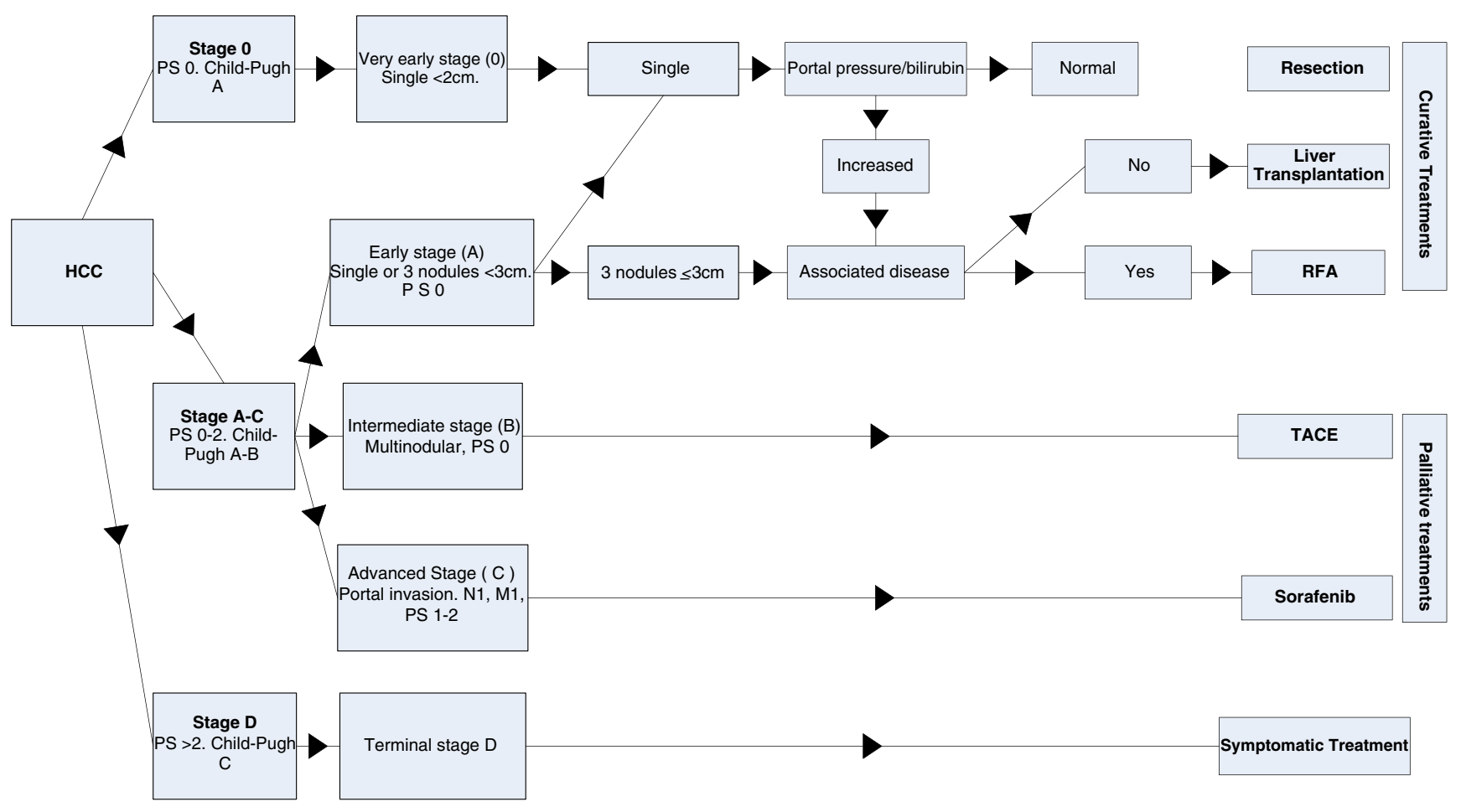

Fig. 1 The BCLC staging system for HCC. $M$ metastasis classification; $N$ node classification; $P S$ performance status; $R F A$ radiofrequency ablation; TACE transarterial chemoembolization 
effects were similar in the two studies and included diarrhea, weight loss, hand-foot skin reaction, and hypophosphatemia. As $\geq 50 \%$ of HCC patients present with advanced disease, continued development of effective systemic therapies for BCLC stages B and C disease may allow an expanded role for complimentary local treatment.

Unfortunately, approximately $10 \%$ to $20 \%$ of patients with $\mathrm{HCC}$ will present with symptomatic, terminal stage, or class $\mathrm{C}$ disease (BCLC stage $\mathrm{D}$ ). Given potential iatrogenic toxicity and the underlying poor functional reserve, these patients are typically considered for symptomatic treatment.

\section{Secondary liver cancer}

Beyond PLC, the liver is a common site of metastasis from multiple primary sites including the gastrointestinal tract, lung, and breast. In addition, certain uncommon malignancies including uveal and cutaneous melanoma, adrenal cortical carcinoma, and some types of sarcoma also have a propensity to metastasize to the liver and may present with this as the only site of disease.

The current management of hepatic metastasis is a product of many decades of research and represents one area in oncology where both local and systemic and treatments have demonstrated therapeutic benefit. Hepatic metastases from colorectal cancer are of substantial oncologic interest in that the liver is often the first site of metastatic disease for colorectal cancer and may constitute the only site of spread in as many as $30-40 \%$ of patients. In this regard, an intermediate state of metastasis [12] termed "oligometastasis" has been proposed [13] and subsequently used to select appropriate patients for aggressive local treatments where an improvement in overall survival is expected from presumed organ confined, isolated metastases treated with local therapies, such as surgery [14-16].

The therapeutic benefit of aggressive local therapy for oligometastatic liver cancer is best exemplified by surgical resection of liver metastases. Similarly, Fong and colleagues [17] reported 5- and 10-year survival rates of $37 \%$ and $22 \%$ among 1,001 consecutive patients undergoing liver resection at Memorial SloanKettering Cancer Center between July 1985 and October 1998 for metastatic colorectal cancer (MCRC). They developed a clinical score for predicting recurrence after hepatic resection for metastatic cancer by assigning one point or based on nodal positivity in the primary, disease-free interval from primary to metastasis, number of hepatic tumors, size of the largest hepatic tumor, and carcinoembryonic (CEA antigen) level exceeding $200 \mathrm{ng} / \mathrm{mL}$. In a subsequent systematic review of 30 studies, Simmonds and colleagues [18] observed that approximately $30 \%$ of patients remained alive 5 years after resection, with median postoperative mortality rate of $2.8 \%$. Additionally, perihepatic abscesses, hepatic failure, generalized sepsis, myocardial infarction, and postoperative hemorrhage and pulmonary embolism represented important sources of morbidity, although these are uncommon in experienced centers. The study corroborated the possibility of extended survival for some patient groups following hepatic resection in the absence of systemic therapy, as evidenced by data by Wei et al., indicating 5-year survival of 48\% among 423 hepatectomies performed for MCRC.

The benefits of hepatic metastasectomy may also extend beyond colorectal cancer patients, although noncolorectal patients have not been extensively studied. In a review of selected case series (with patient numbers ranging from 17 to 65 highlighting the relative lack of clinical experience), Elias et al. [19] reported 5-year survival rates of $16 \%$ to $61 \%$ for patients with liver metastases from breast cancer treated with hepatic resection, highlighting the complexities in evaluating the therapeutic benefit for breast cancer, given the disease heterogeneity, particularly with respect to hormone receptor status, and continued improvement in systemic regimens.

\section{Rationale for radiation for primary and metastatic liver cancers}

As highlighted in the prior discussion, local therapy plays a significant role in the treatment of both HCC and liver metastases, establishing a potential rationale for radiation therapy for unresectable patients. As summarized by Dawson et al. [20], potential RT candidates range from very early- and/or early-stage tumors in medically inoperable patients or as bridge therapy for those with HCC awaiting OLT, particularly when RFA is contraindicated. In intermediate and advanced-stage patients, definitive radiation is suitable for patients who are unfit or refractory to TACE and as potential adjuncts to systemic treatments such as sorafenib for stage $\mathrm{C}$ patients. In addition, stage $\mathrm{D}$ patients may derive palliative benefit from short-course irradiation.

Similarly, among patients with liver metastasis, radiation therapy may also provide long-term control, particularly for patients with three or fewer tumors $\leq 3 \mathrm{~cm}$ in diameter and in the absence of extrahepatic disease. While prospective trials elucidating the role of radiation therapy are emerging, the role of RT in liver cancer has not been established through level I evidence (a RTOG phase III trial comparing sorafenib alone vs RT followed by sorafenib is being planned in locally advanced HCC patients). It is our objective in the remaining sections to provide an overview of the imaging modalities, and RT techniques, toxicity, fractionation 
schemes, and their efficacy in the management of primary and metastatic liver cancers.

\section{Radiotherapeutic imaging of liver tumors}

Hepatic anatomy poses unique challenges which necessitate high-quality imaging both for diagnosis and treatment. Unlike the brain and spinal cord, which are constrained by bony structures, the liver's location in the abdominal cavity allows for significant displacement and deformation, as a result to intrinsic and extrinsic changes. Anatomically, the liver is divided into eight segments using the Couinaud classification [21], following the major divisions of the bile duct, portal venous, and arterial structures. In addition, the liver is surrounded by multiple soft tissue structures including kidneys, spinal cord, duodenum, stomach, and large bowel.

From an RT perspective, computed tomography (CT) represents the cornerstone of hepatic imaging, as it provides undistorted, fast data acquisition over the entire abdominal cavity. Using current multidetector CT scanning, the entire liver can be imaged in one pass within a single breath-hold. Importantly, through the use of Hounsfield data, CT also provides a convenient method through which tissue heterogeneities can be incorporated in modern treatment planning. High-quality contrast-enhanced CT scanning allows the capture of distinct phases including the arterial, portal, and venous phases with the ability to provide multidimensional reconstruction. CT also allows excellent imaging of vascular anatomy but remains limited in its soft tissue contrast.

Similar to CT scan, magnetic resonance (MR) imaging provides comprehensive and accurate multi-dimensional information concerning hepatic lesions, parenchyma, and adjacent organs. A particular strength is its ability to vary intrinsic soft tissue characteristics and soft tissue contrast. Specifically, T1-weighted imaging is useful for detecting small, fat-containing lesions. T2-weighted imaging allows distinction between solid and non-solid components based on fluid content, and dynamic contrast enhancement permits lesion detection and characterization with contrast media permitting distinction between primary and secondary liver lesions. At present, the clinical impact of $3 \mathrm{~T}$ on hepatic imaging is controversial.

Beyond the diagnostic considerations, imaging for radiation oncology has special requirements including the need for larger bore size to accommodate immobilization devices, a flat instead of a horizontal couch, special immobilization devices, and, perhaps most importantly, spatial accuracy. In this regard, a prospective study was designed at the University of Toronto [22] to compare CT and MR imaging for liver cancer gross tumor volume (GTV) delineation using deformable image registration. The study included 26 patients with unresectable liver cancer, among them eight with liver metastasis, ten with HCC, and eight with intrahepatic cholangiocarcinoma. The GTV was defined on IV contrast planning CT with clinical target volume (CTV) constructed by adding an individualized $0.8 \mathrm{~cm}$ margin. As expected, for CT scan imaging, the arterial phase provided the optimal imaging for seven out of ten HCCs, and the venous phase was preferred for six of eight cholangiocarcinomas and five of eight metastases. In contrast, optimal magnetic resonance imaging (MRI) delineation sequences were variable. Even with the use of deformable registration, significant differences were seen in a number of tumor foci with median distance between the $\mathrm{CT}$ and MR tumor surface of $3.7 \mathrm{~mm}$ and significant percentage of tumor surface differing by $\geq 5 \mathrm{~mm}$ of $26 \%$. Concordance volumes ranged from $64 \%$ in cholangiocarcinoma to $81 \%$ in metastasis, demonstrating clinically relevant modality-dependent differences in target volumes.

\section{Pathologic principles of liver RT}

The successful ability to safely deliver radiobiologically potent radiation dosages requires a thorough understanding of dose-volume tolerances of both the liver and surrounding critical structures, in addition to the pathogenesis of radiation-induced liver disease (RILD).

Prior to the 1960 s, the liver was considered radioresistant, although subsequent case reports began to describe parenchymal necrosis and fibrosis. Subsequent studies performed at Stanford University examined incidentally irradiated livers due to treatment of ovarian carcinoma, lymphoma, or esophageal cancer. The histologic findings included hyperemia, hepatic cell loss, vein lesions, parenchymal fibrosis, and hyperplasia [23]. The central and sublobular veins exhibited occlusive changes, and the pathologic changes were most pronounced near the lobular centers and associated with progressive obliteration of small branches of the hepatic veins. Subsequent work by Fajardo et al. [24] further characterized veno-occlusive disease (VOD). The pathologic findings were clinically associated with weight gain, increasing abdominal girth, hepatomegaly and ascites, jaundice, and elevation of liver enzymes (particularly alkaline phosphatase). RT liver injury was further characterized by Lawrence et al. [25] as severe congestion of sinusoids in the central portion of the lobules with atrophy of the inner portion of the liver plates and absence of liver cells around the central veins. The changes activate the coagulation cascade, leading to the accumulation of fibrin and formation of clots in central veins and hepatic sinusoids with subsequent erythrocyte trapping, vascular congestion, and decreased oxygen delivery to the central zone, resulting in death of centrilobular hepatocytes and atrophy of the inner hepatic plate [26]. 
Management of radiation-induced liver toxicity centers on supportive care (diuretics for fluid retention, analgesics, and steroids). Antiviral therapy for the hepatitis B viral (HBV) carriers has also been advocated to prevent exacerbation [27]. Uncontrolled trials have also utilized anticoagulation, glutathione selenium, and vitamin E, with a more recent potential mitigator, defibrotide, a polydisperse oligonucleotide with antithrombotic properties. A phase II randomized trial [28] demonstrated efficacy in stem cell transplant patients with severe VOD, and preliminary reports from phase III trials are encouraging [29].

\section{Clinical evolution of radiation therapy in liver cancer}

Given the aforementioned recognition of RILD, and as a reflection of limited technology and understanding of partial organ dose-volume concepts, early efforts in liver irradiation focused on whole liver RT (WLI). Borgelt et al. [30] subsequently described the results of a prospective RTOG study for liver metastasis. Symptomatic improvement ranged from 19\% to $55 \%$, with performance status improvement in $25 \%$. A subsequent protocol investigated WLI to 27,30 , and 33 Gy at $1.5 \mathrm{~Gy} /$ fraction twice daily [31] and failed to demonstrate improvement in median survival but revealed an important dose-volume toxicity effect. Radiation-induced liver injury was absent among patients receiving 27 or $30 \mathrm{~Gy}$, but rose to $10 \%$ risk at 6 months for patients receiving $33 \mathrm{~Gy}$. Altogether, the clinical data on WLI (summarized in Table 1) (a) demonstrates the palliative potential of WLI for patients who are not suitable for alternative therapies and (b) establishes the tolerance dose for WLI at approximately 30 Gy [32]. The studies also revealed that, for patients with better prognosis including oligometastatic disease, WLI is of limited effectiveness.

\section{DCRT and dose-volume relationship for liver toxicity}

Our current understanding of partial liver tolerance doses is based to a large extent on the prospective series of trials conducted at the University of Michigan (UM). These studies utilized three-dimensional conformal radiation therapy (3DCRT) planning and concurrent chemosensitization, initially with fluorodeoxyuridine (FUdR) and later bromodeoxyuridine (BrdU) through hepatic arterial infusion. In a phase I trial, Robertson et al. [33] utilized HA BrdU with doses (24-66 Gy at 1.5 Gy BID) as a function of the fraction of normal liver treated. Results revealed subacute or longterm complications in four patients, including duodenal ulcers in two patients and one case of RILD, highlighting the critical radiosensitivity of the small bowel as a doselimiting organ. Subsequent trials from UM employed doseescalated radiotherapy, using prescription doses as a function of predicted Lyman normal tissue complication probability (NTCP) modeling [34]. This metric utilizes three parameters: (1) TD50, defined as the whole liver dose associated with $50 \%$ probability of toxicity; (2) $m$, characterizing the steepness of dose-response at TD50; and (3) $n$, a volume effect parameter, which indicates larger volume effect as it increases. The model parameters were initially fit using clinical data from 71 patients treated with 3DCRT, out of which nine developed clinical radiation hepatitis [35]. Notably, patients who developed RT toxicity had received WLI, with mean liver doses $\geq 37$ Gy. The data supported dose escalation, as radiation dose correlated with improved survival. A subsequent update of the UM experience [36] described an updated NTCP model analysis among 203 patients, among which 19 developed RILD. Revised Lyman model parameters among patients receiving FUdR were: (1) TD50, 45.8 and 39.8 Gy for metastases and PLC, respectively; (2), $m, 0.12$; and (3) $n, 0.97$. No cases of RILD occurred with mean doses $\leq 31$ Gy. Additional toxicity predictors included primary hepatobiliary cancer versus metastasis, BrdU chemotherapy, and male gender. TD5 RILD levels for metastatic and primary liver tumors were predicted at mean liver doses of 32 and 28 Gy, respectively, using 2 Gy fractions for patients with Child-Pugh class A liver function [37]. Of note, the UM parameters must be interpreted with caution given the BID fractionation, $1.5 \mathrm{~Gy}$ fraction size, and the absence of Child-Pugh class B patients [38]. Additional investigation is needed to develop specific model parameters, and alternative models to account for worse underlying hepatic disease, alternative fractionations, and more inclusive toxicity endpoints than classic RILD.

Other groups have demonstrated increased biologic susceptibility to RILD among HCC patients and have defined
Table 1 Results of palliative whole-liver RT for liver metastases

\begin{tabular}{llll}
\hline & $N$ & Palliative relief (\%) & OS (m) \\
\hline Sherman et al. [89] & 55 & 90 & 4.5 \\
Sherman et al. (responders) [89] & 21 & - & 9.0 \\
Borgelt et al. [30] & 103 & 19 , Fatigue & 3.75 \\
Mohiuddin et al. [90] & 33 & 55, Pain & 4 \\
Mohiuddin et al. (Partial Boost) [90] & 12 & 100, Pain & 14 \\
\hline
\end{tabular}


"non-classic" RILD [39] empirically as $\geq 5$-fold elevation of transaminases relative to pretreatment. In a cohort of 89 patients, Cheng et al. [40] observed 17 cases of RILD, with reactivation of viral hepatitis B during RILD. Chronic HBV carrier status and mean liver dose were also associated with RILD. Of note, the mechanism for radiation-induced hepatitis B virus reactivation has been further investigated and linked to bystander effects on irradiated endothelial cells releasing cytokines including IL-6 [41]. Tolerance data relevant to hypofractionated schemes are presented in a subsequent section of this document.

\section{Special clinical indication for radiotherapy in HCC}

A number of clinical scenarios arise in patient care for which refinement in current standard management is evolving, with potential for increased radiotherapy role. Some of the scenarios with a strong opportunity for radiation therapy to improve outcomes are described below. A representative case involves PLC treatment in the setting of inferior vena cava tumor thrombus (IVCTT), which occurs in approximately $4 \%$ of patients at initial presentation or following repeated TACE. This clinical condition is associated with increased risk of sudden death due to heart failure or pulmonary embolism, and prognosis remains poor. Treatment options are limited to surgical removal of the tumor thrombus, which is often contraindicated due to diminished hepatic reserve. Given historical poor outcomes with TACE alone in this clinical setting, Koo et al. [42] retrospectively compared a cohort of patients who underwent TACE and 3DCRT (median dose of $45 \mathrm{~Gy}$ ) to 29 historical patients receiving TACE alone. Results demonstrated significant improvement in response and progression-free rates of IVCTT using the combined treatment, with improved median survival times of 11.7 versus 4.7 months, respectively.

RT has also proven effective in treatment of portal venous thrombosis (PVT), another more common condition demanding attention and for which standard therapies have historically proven suboptimal. Huang et al. [43] retrospectively reviewed their experience with 326 patients with imaging-diagnosed PVT treated with 60 Gy in 20-30 fractions. Objective responses were achieved in approximately $25 \%$, with improved survival observed in responders (13.3 and 11.6 months in complete and partial responders vs 4.5 months in nonresponders). The data suggested a doseresponse at $50 \mathrm{~Gy}$, with suggestion of improved efficacy for patients with ECOG performance status of 1-2.

It is estimated that approximately $20 \%$ of patients with advanced HCC listed for liver transplantation are delisted as a result of local tumor progression. In an attempt to minimize this occurrence, RT has been proposed, as a potential bridge strategy, particularly in patients unsuited for more conventional therapies including TACE. A number of case reports have begun to emerge [44]. Sandroussi et al. [45] recently reported their experience with ten patients treated at University of Toronto, with failed prior local therapies or unsuitability for given poor liver function or anatomic constraints (with HCC beyond Milan criteria). Irradiation volumes and doses were individualized to spare involved liver and critical structures. Median RT dose was $33 \mathrm{~Gy}$ in one to six fractions, and nine of ten patients completed RT as planned (one was unexpectedly called for a liver transplant after only one fraction). With a median follow-up of 14 months, local tumor control was achieved in all treated tumors, with two patients delisted as a result of out-of-field progression. Five patients underwent transplant without unforeseen complications. Explant pathology revealed tumor necrosis ranging from $40 \%$ to $90 \%$ with a suggestion of increased intratumoral fibrosis among those patients receiving prior TACE. The authors concluded that 3DCRT represents a safe and effective transplant bridging therapy for selected HCC patients awaiting liver transplant.

\section{Future directions and opportunities in liver radiation oncology}

Management of unresectable, large-volume HCC continues to represent a significant therapeutic challenge. In this regard, a number of groups have demonstrated improved clinical response rates and survival when combining TACE with radiation therapy [46, 47]. Meng et al. [48] recently performed a systematic review of 17 trials comparing TACE alone versus combined TACE and RT in unresectable HCC. Results demonstrated that combined TACE-RT was associated with improved survival at 1 year (odds ratio, 0.23 ). Statistically significant benefits persisted at the 2-, 3-, and 5year survival endpoints.

Prospective studies are also emerging which describe high-dose radiation as a potentially curative treatment of unresectable HCC. Among these, a French phase II trial $[49,50]$ utilized high-dose 3DCRT of up to $66 \mathrm{~Gy}$ in 33 fractions for low-volume HCC and, among 27 patients, reported objective and complete responses in $92 \%$ and $80 \%$ of patients, respectively, with grade 4 toxicities only occurring among Child-Pugh class B patients (2 of 11). At a median follow-up of 29 months, $22 \%$ and $41 \%$ of patients developed in-field versus out-of-field recurrences. Overall, the results demonstrate encouraging efficacy for patients with Child-Pugh class A disease, with a cautionary note when treating Child-Pugh class B cirrhotic patients, a patient subset that may benefit from particle beam treatment. Hata et al. [51] reported on 19 HCC patients with ChildPugh class C cirrhosis treated to a median of 72 Gy in 10-22 fractions. Results demonstrated an objective response rate of 
$63 \%$ with progression-free survival of $91 \%$ at 17 months and disease-free survival of $31 \%$ at 2 years. Importantly, no grade 3 or 4 early or late toxicities were described.

Recent reviews and studies have attempted to combine sorafenib with radiation for unresectable HCC. The rationale for this combination stems from targeting of molecular pathways including the Ras, Raf, MAP-K, and VEGFR signaling pathways, which are activated after radiation exposure and may be responsible for radioresistance [52]. While promising, this strategy needs close monitoring of potential unexpected toxicities occurring at doses lower than expected, including radiation dermatitis or gastrointestinal luminal toxicity such as GI bleeds [53].

\section{Technical aspects of image-guided radiotherapy and SBRT}

Therapeutic radiation for liver malignancies has benefited from progressive advances in imaging, target delineation, and treatment planning methods enabling efficacy and toxicity studies. Dose-volume constraints for conventional and hypofractionated approaches have emerged (Table 2). Technically, successful RT delivery to progressively small volumes has culminated in SBRT, for which treatment planning and delivery are particularly challenging for liver cancers. SBRT may be defined as a precise therapeutic modality delivering high radiation doses to an extracranial target using either a single or small number of fractions [54]. SBRT planning and delivery evolved from intracranial stereotactic radiosurgery (SRS), with principles now dating close to half a century to the development of the Gamma Knife at the Karolinska Institutet in Sweden. In the brain, SRS is simplified by the ability to perform rigid fixation of the skull to an external rigid frame and creation of a stereotactic coordinate system. For extracranial sites, this technology has been very successfully applied to lung tumors and has been tested successfully in phase II studies. Comparatively, lung SBRT is also less challenging than its liver counterpart given superior imaging due to high soft tissue contrast from lung to soft tissue tumor densities allowing for straightforward verification using standard kilo- and megavoltage volumetric imaging systems at the treatment unit. In this respect, liver SBRT, given a lack of inherent contrast between the tumor and normal liver and substantial respiration-induced motion, presents substantial challenges for imaging and RT treatment planning [55]. In this section, technical parameters relating to photon-based, highprecision conformal and SBRT for liver malignancies will be discussed.

In view of high potential for target underdosage and overdosing of critical structures, SBRT necessitates enhanced precision in all aspects of RT planning and delivery (i.e., immobilization and image acquisition, treatment planning, and dose delivery).

\section{Simulation and target delineation for high-precision liver RT}

Initial attempts at liver SBRT utilized stereotactic body immobilization with a body frame or similar devices, which attempted to translate tumor position to a rigid coordinate system as customary for intracranial frame-based SRS. Such approaches predated the use of image guidance and, in most clinics, were superseded by the use of vacuum bags with or without abdominal compression and breath-hold imaging. Frameless platforms require four-dimensional volumetric imaging, which is typically based on high-quality contrastenhanced CT. Nevertheless, the liver is subject to significant deformation due to differences in image acquisition protocols and different patient support couches between diagnostic and treatment units (i.e., rounded versus flat couch tops). In an effort to optimize image acquisition and therefore minimize the expansion margins owing to potentially suboptimal image registration, contrast-enhanced, liverspecific, four-dimensional (4D) CT scanning has been advocated by multiple groups $[55,56]$. The contrast injection

Table 2 Dose-volume limits for liver and adjacent organs (conventional fractionation) (adapted from (Emami et al. [32])

\begin{tabular}{|c|c|c|c|}
\hline & Liver metastases & Primary liver cancer & Comment \\
\hline Whole-liver RT & $\leq 30 \mathrm{~Gy}, 2 \mathrm{~Gy} / \mathrm{F} 21 \mathrm{~Gy} / 7 \mathrm{~F}$ & $\leq 28 \mathrm{~Gy}, 2 \mathrm{~Gy} / \mathrm{F} 21 \mathrm{~Gy} / 7 \mathrm{~F}$ & Whole-organ prescription dose \\
\hline $\begin{array}{l}\text { Partial-liver RT, conventional } \\
\text { fraction }\end{array}$ & $\leq 32 \mathrm{~Gy}$ & $\leq 28 \mathrm{~Gy}$ & $\begin{array}{l}\text { Mean normal liver }{ }^{\mathrm{a}} \text { dose for tumor dose } \\
\leq 2 \mathrm{~Gy} / \mathrm{F}\end{array}$ \\
\hline SBRT, 3-6 F & $<15 \mathrm{~Gy} / 3 \mathrm{~F}<20 \mathrm{~Gy} / 6 \mathrm{~F}$ & $\begin{array}{l}<13 \mathrm{~Gy} / 3 \mathrm{~F}<18 \mathrm{~Gy} / 6 \mathrm{FCP}(\mathrm{B}) \\
<6 \mathrm{~Gy} / 4-6 \mathrm{~F}\end{array}$ & Mean normal liver ${ }^{\mathrm{a}}$ dose \\
\hline$<15 \mathrm{~Gy} / 3 \mathrm{~F}$ at least $800 \mathrm{~mL}$ & $\begin{array}{l}\geq 700 \mathrm{~mL} \text { normal liver } \\
<15 \mathrm{~Gy} / 3 \mathrm{~F}\end{array}$ & $\geq 800 \mathrm{~mL}$ normal liver $<18 \mathrm{~Gy} / 3 \mathrm{~F}$ & $\begin{array}{l}\text { Critical volume model only for Child-Pugh } \\
\text { class A }\end{array}$ \\
\hline
\end{tabular}

Modified with permission [17]

$F$ fraction, GTV gross tumor volume, $C P$ Child-Pugh class

${ }^{a}$ Normal liver refers to the total volume of liver minus the gross tumor volume 
typically involves $150 \mathrm{~mL}$ injected at a flow rate of $5 \mathrm{~mL} / \mathrm{s}$, although weight-based dosing is increasingly incorporated at some centers. 4D Imaging is acquired in the delayed phase $(>180 \mathrm{~s})$ to avoid variability between arterial (20$30 \mathrm{~s}$ ) and venous (50-60 s) phases, and providing optimal imaging for metastatic cases with more difficult visualization of HCC. Due to blurring effect, image quality is typically inferior compared with a diagnostic scan. For calculation sequences, either the average or breath-hold sequences, but not free-breathing images, are recommended by the authors. Alternatively, as proposed by Brock et al. [54, 55], breath-hold image acquisition can also be performed for target delineation. Newer CTs can further optimize the timing of contrast enhancement for imaging purposes with automated triggering, and image fusion of the primary dataset to MRI and/or PET-CT may aid in GTV delineation. Substantial variation still exists in CTV and planning target volume (PTV) margins. Emerging experimental evidence demonstrates microscopic extension from the GTV does exist in hepatic cancers and metastatic tumors. For example, among 149 resected HCC patients, clinicopathologic parameters including tumor volume, presence of portal vein thrombosis, and elevated AFP were utilized to predict microinvasion in patients with HCC [57]. Additionally, among 100 patients with intrahepatic cholangiocarcinoma (IHC) undergoing resection, Bi et al. [58] identified microinvasion ranging from 0.4 to $8 \mathrm{~mm}$ in $65 \%$ of patients. A scoring system was devised from tumor boundary type, TNM stage, grade, CA19-9, ALT, AST, GGT, and alkaline phosphatase. More recently, clinicopathologic correlation for colorectal liver metastases was performed in 13 patients with 21 colorectal liver metastases who underwent pre- and postoperative MRI. Volumetric analysis was performed and revealed good agreement with the pathologic findings, with microscopic extension between 0.2 and $1.0 \mathrm{~cm}$ from the main tumor. Microscopic extension is small and may be contained within the tumor capsule or enhancement zone as visualized in imaging modalities. In practice, the authors recommend no additional margin beyond the imaging-defined enhancing tumor. Different institutions currently utilize GTV to CTV expansions of 0 to $1 \mathrm{~cm}$ [59].

\section{Motion management and image guidance in liver RT}

A number of strategies have been devised for incorporation of respiratory motion for abdominal targets. These are summarized in the AAPM Task Group (TG) report 76 [60] and, more recently, for liver cancer-specific applications by Brock et al. [55]. TG-76 stipulates respiratory motion management when (a) available, if (b) target motion exceeds $5 \mathrm{~mm}$, and (c) when motion management is tolerable by the patient. An algorithm for CT-based treatment planning of abdominal targets developed by Balter et al. uses static exhale images [61]. In their study, CT scans were acquired at normal exhalation and margins placed based on the ventilatory excursion inferior to the target. Measurements revealed that the diaphragm remained within $25 \%$ of the range of the ventilatory excursion for $42 \%$ of the typical breathing cycle. The reproducibility of exhale positioning over multiple breathing cycles was $0.9 \mathrm{~mm}$, leading to typical margins of $1.0 \mathrm{~cm}$ superior to the target and $1.9 \mathrm{~mm}$ inferior to the target, with a $4 \%$ reduction in liver $V_{\text {eff. }}$ Practical motion management strategies also include motion reduction via abdominal compression, breath-hold techniques, and gating and tracking methods. Advantages of simpler methods such as abdominal compression include improved duty cycle (i.e., percentage of time that the patient is receiving treatment relative to total time in the treatment unit), simplicity, and improved patient comfort, at the expense of proportional gains in reduction of irradiated treatment volumes with more sophisticated methods including breath-hold and gating and tracking techniques. Emerging clinical studies have begun to quantify the residual treatment errors including residual uncertainties and deformation to optimize motion management. In an evaluation of 83 conebeam CT (CBCT) scans from 16 patients with $30 \mathrm{GTVs}$, Eccles et al. [62] observed small deformations due to abdominal compression among patients undergoing SBRT.

At present, most clinics evaluate organ motion by means of a treatment planning 4DCT, and therefore potential exists for intra- and interfractional changes in liver motion patterns relative to treatment planning. Fortunately, research evidence suggests that these changes are within clinical tolerance, and usually, there is less motion as the treatment course goes on. In a cohort of 29 patients undergoing liver SBRT with or without abdominal compression, Case et al. [63] demonstrated small $(\leq 2 \mathrm{~mm})$ absolute inter- and intrafraction amplitude changes. In contrast, the same group [64] found larger interfraction liver position changes $(2.9 \pm$ $1.1 \mathrm{~mm}$ ) for non-breath-hold liver SBRT, providing a rationale for soft tissue (i.e., liver) rather than bony anatomybased image guidance. Liver positional reproducibility has also been evaluated for active breathing coordinator (ABC) breath-hold treatment, considered useful for patients with average free breathing motion ranges exceeding $0.5 \mathrm{~cm}$. Among 21 patients, Eccles et al. [65] confirmed diaphragmatic stability using fluoroscopy, with mean intra- and interfraction CC offsets in diaphragmatic position related to the vertebral bodies of 1.7 and $3.7 \mathrm{~mm}$, respectively. Newer motion management technologies include beam gating and target tracking and require synchronization between the detection of the tumor or its target and the linear accelerator. These technologies are under active investigation, but for liver applications, specifically, their reproducibility is 
less well quantified compared with breath-hold and abdominal compression techniques.

A number of image guidance and motion reduction techniques have been developed to minimize tumor motion and, therefore, PTV margins during liver SBRT. For image guidance using two-dimensional orthogonal MV imaging (perhaps the simplest IGRT approach), the vertebral bodies can be identified and used to guide mediolateral and anteroposterior position of the patient, while the diaphragm can be used for CC positioning of the patients (since CC shifts in liver may occur relative to the vertebral body). When combined with breath-hold technology, the residual errors following MV imaging using these surrogates were estimated at $\leq 5 \mathrm{~mm}$ in each direction [66]. The same authors demonstrated improved setup reproducibility using KV CBCT alignment to the planning $\mathrm{CT}$ with average positional differences of $0.2,0.6$, and $0.0 \mathrm{~mm}$ in the $\mathrm{CC}$, AP, and $\mathrm{ML}$ dimensions. In addition, the average residual deformation of the liver following rigid registration of the liver on repeat volumetric imaging was small, with the average of $95 \%$ of the liver volume deforming by less than $2.3 \mathrm{~mm}$. Notable exceptions included the dome of the liver, medial liver, and the inferior liver tip, where more than $5 \%$ of the liver deformed by more than $5 \mathrm{~mm}$ in cases.

In cases where volumetric image guidance is not available, percutaneous radiopaque markers can be implanted. Kothary et al. [67] analyzed 34 fiducial marker implantations in the liver to aid in patient positioning. Major and minor complication rates were estimated at $5 \%$ and $17.3 \%$, respectively. Marker migration was documented in $4.3 \%$ cases; in this regard, migration of an implanted marker to the inferior vena cava requiring extraction through angiography has been reported [68].

Continued improvements are expected in abdominal radiotherapy as investigators further enhance imaging and dose-calculation algorithms (i.e., four-dimensional and Monte Carlo calculation methods, for example). Likewise, given that conventional three-dimensional CBCT is subjective substantial blurring artifact, retrospective sorting of respiratory-correlated CBCT (i.e., 4D-CBCT) [69] significantly reduces artifacts and is now being clinically implemented.

\section{Development of SBRT for liver tumors}

The previously described technical advances in treatment planning, dose delivery, and image guidance coupled with 3DCRT data describing enhanced partial volume irradiation tolerances permitted formulation of dose-response relationships for liver tumors. A report by Park et al. [70] identified total dose as the most significant associated with tumor response and demonstrated dose-dependent response and toxicity rates. Subsequently, Park et al. [71, 72] corroborated the dose-response data. With a threshold of $50 \mathrm{~Gy}_{10}$ (using 2-3 Gy fractions), the higher response rates attained at higher doses correlated with overall survival at 2 years following RT. Based on these developments and the aforementioned clinical data on partial liver tolerance from UM among other centers, and given technological improvements allowing extrapolation of extra-cranial radiotherapy, SBRT for liver tumors has gathered significant clinical interest during the past two decades.

Initial reports were largely descriptive and included patients with both primary and secondary liver cancers. More comprehensive studies have subsequently emerged. Blomgren et al. [73] reported on one of the first studies for SBRT to lung, liver, or retroperitoneal tumors, and described objective response rates of $50 \%$. The technique borrowed from intracranial radiosurgery and delivered a planned inhomogeneous dose to the PTV. Subsequently, Herfarth et al. [74] presented data for (predominantly) metastatic liver patients treated with single fraction SBRT to 14 26 Gy and described actuarial local control of $81 \%$ at 18 months. Likewise, Wulf et al. [75] described SBRT for cohort of primary liver cancers and hepatic metastases. Their study used a planned inhomogeneous boost by using a prescription isodose line of $65 \%$ to cover the PTV. Prescription doses range from $3 \times 10$ to $3 \times 12.5$ Gy. Results indicated local control of $100 \%$ for five patients with primary liver cancer and $82 \%$ for 39 patients with 51 hepatic metastases at a median follow-up of 15 months. Of note, local control was significantly improved among patients with prescription doses of $3 \times 12.5$ or $1 \times 26$ Gy. More recently, a number of investigators have reported on primary liver-cancer-specific trials (Table 3) [76].

Reflective of the current improvements in treatment planning and dose-delivery methods, single-fraction SBRT has recently been studied using the Cyberknife robotic system. Goodman et al. [77] reported on 26 patients treated for 40 radiated lesions, both with hepatic metastases and primary liver tumors with dose escalation from 18 to 30 Gy using single-fraction SBRT. The authors described good tolerance and absence of dose-limiting toxicities with $77 \%$ local control rate at 17 months median follow-up and with overall survival of 29 months. Similarly, Goyal et al. [78] reported on 17 patients (nine PLC, eight metastatic) receiving 34 Gy over 1-3 fractions as SBRT with local control of $82 \%$.

\section{Recent experience with SBRT for PLC}

With respect to primary liver tumors, Kwon et al. [79] reported on 42 unresectable Child-Pugh class A HCC patients treated with SBRT to 30-39 Gy in three fractions (mean tumor volume of $15.4 \mathrm{cc}$ ). Results demonstrated 
Table 3 Results of SBRT for primary liver cancer

\begin{tabular}{|c|c|c|c|c|c|c|}
\hline & $N$ & Median FU (m) & Dose (Gy) & RR (\%) & LC $(\%)$ & OS $(\%$ or $\mathrm{m})$ \\
\hline Kwon et al. [79] & 42 & 28.7 & $10-13 \times 3 \mathrm{fx}$ & 85.8 & $\begin{array}{l}72 \text { at } 12 \mathrm{~m} \\
68 \text { at } 36 \mathrm{~m}\end{array}$ & $\begin{array}{l}93 \% \text { at } 12 \mathrm{~m} \\
59 \% \text { at } 36 \mathrm{~m}\end{array}$ \\
\hline Cardenes et al. [81] & 25 & 24 & $\begin{array}{l}12-16 \times 3 \mathrm{fx}(\mathrm{CPA}) \\
8 \times 5 \mathrm{fx}(\mathrm{CPB})\end{array}$ & - & 100 & $\begin{array}{l}75 \% \text { at } 12 \mathrm{~m} \\
60 \% \text { at } 24 \mathrm{~m}\end{array}$ \\
\hline Facciuto et al. [82] & 39 & 22 (post-SBRT) & $4 \times 7$ (median) & $\begin{array}{l}37 \text { (clinical) } \\
37 \text { (pathologic) }\end{array}$ & & $\begin{array}{l}32 \mathrm{~m} \text { (OLT) } \\
14 \mathrm{~m} \text { (no OLT) }\end{array}$ \\
\hline Tse et al. [76] & 41 & 17.6 & 24-54 (median, 36) & 49 (RECIST) & 65 at $12 \mathrm{~m}$ & $\begin{array}{l}\mathrm{HCC}, 11.7 \mathrm{~m} \\
\mathrm{IHC}, 15.0 \mathrm{~m}\end{array}$ \\
\hline
\end{tabular}

$85.8 \%$ complete or partial response rates with mean time to CR or PR of 5.1 months. In-field progression was documented in $29 \%$ of patients, but all 25 patients who achieved complete response maintained it throughout the follow-up duration. Hepatic out-of-field progression occurred in 18 patients and distant metastasis in 12 patients $(42.9 \%$ and $28.6 \%$ ), respectively. Tumor volume significantly predicted progression-free survival rate at 1 year. Additional analysis from that institution [80] evaluated dosimetric predictors of hepatotoxicity and observed 33\% incidence of grade II or higher toxicity, with an $11 \%$ probability of progression of $\mathrm{CP}$ class. The hepatic liver volume receiving $\leq 18 \mathrm{~Gy}$ emerged as the only significant predictor of progression of CP class using three-fraction SBRT, and the authors recommended a minimum $800 \mathrm{cc}$ of liver should receive $\leq 18 \mathrm{~Gy}$ to prevent the risk of deterioration of hepatic function.

A phase I dose escalation trial was recently conducted by Cárdenes [81] at the University of Indiana. This was a dose escalation study including patients with one to three lesions and $\mathrm{CP}$ class $\mathrm{A}-\mathrm{B}$ inoperable patients with tumors $\leq 6 \mathrm{~cm}$. Dose escalation was performed uneventfully among two patients with Child-Pugh B disease developing grade III hepatic toxicity at 42 Gy requiring subsequent amendment of these patients to receive five fractions. Six patients underwent liver transplant and ten patients described as alive without progression at in follow-up of 24 months with overall survival rate of $75 \%$ at 1 year.

Facciuto et al. [82] provided excellent clinicopathologic correlation among 39 lesions in 27 patients receiving SBRT as bridge therapy prior to OLT. Patients received 24-36 Gy divided in two to four fractions using Novalis-based radiosurgery with image guidance and fiducial marker placement. Result demonstrated a $37 \%$ clinical complete response rate with pathologic complete and partial response rates of $14 \%$ and $23 \%$. Post-OLT survival among irradiated patients was similar to that of their transplanted cohort during the same period not receiving SBRT, with post-SBRT median survival of 32 months. Ten non-transplanted patients had median post-SBRT survival of 14 months. The authors concluded that SBRT could be a potential adjunct to standard bridging transplant treatments including RFA, TACE, and radioactive spheres, with optimal dose, fractionation, and timing to transplant needing further evaluation.

\section{Recent experience with SBRT for secondary liver tumors}

Given the potential long-term survival following resection of hepatic metastases from colorectal carcinoma, but the limited

Table 4 Results of SBRT for liver metastases

\begin{tabular}{|c|c|c|c|c|c|c|}
\hline & $N$ & Median FU (m) & Dose (Gy) & RR (\%) & LC $(\%)$ & OS $(\%$ or $\mathrm{m})$ \\
\hline Herfarth et al. [74] & 56 & 5.7 & $14-26 / 1 \mathrm{fx}$ & 90 & 81 at $18 \mathrm{M}$ & \\
\hline Wulf et al. [75] & 51 & 15 & $\begin{array}{l}10 \times 3 \\
12-12.5 \times 3\end{array}$ & - & 82 (crude) & -0 \\
\hline Hoyer et al. [83] & 44 (liver) & 52 & $15 \times 3$ & - & 86 at $24 \mathrm{~m}$ & $38 \%$ at $24 \mathrm{~m}$ \\
\hline Rusthoven et al. [84] & 63 & 16 & $12-20 \times 3$ & - & 92 at $24 \mathrm{~m}$ & $20.5 \mathrm{~m}$ \\
\hline Lee et al. [85] & 68 & 10.8 & Individualized median $41.4 / 6 \mathrm{fx}$ & 49 & 71 at $12 \mathrm{~m}$ & $17.6 \mathrm{~m}$ \\
\hline Rule et al. [86] & 27 & 20 & $\begin{array}{l}10 \times 3 \\
10-12 \times 5\end{array}$ & $\begin{array}{l}90 \text { (60 Gy) } \\
50 \text { (50 Gy) } \\
30 \text { (30 Gy) }\end{array}$ & $\begin{array}{l}100 \text { at } 24 \mathrm{~m} \\
(60 \mathrm{~Gy})\end{array}$ & $37 \mathrm{~m}$ \\
\hline Van der Pool et al. [87] & 20 & 22 & $12.5-15 \times 3$ & - & 74 at $24 \mathrm{~m}$ & $83 \%$ at $24 \mathrm{~m}$ \\
\hline
\end{tabular}


owing to unresectability in a substantial proportion of patients, a number of investigators have conducted clinical trials of SBRT for patients with CRC with liver metastases (Table 4). Hoyer et al. [83] described a phase II trial of SBRT for pre- dominantly hepatic, unresectable CRC metastases and reported 2 -year local or distant progression-free survival rates of $19 \%$ with overall survival of $38 \%$. The prescribed dose was $45 \mathrm{~Gy}$ to the isocenter in three fractions.
Fig. 2 a Pre-SBRT. b Planning. c Post-SBRT

\section{a}
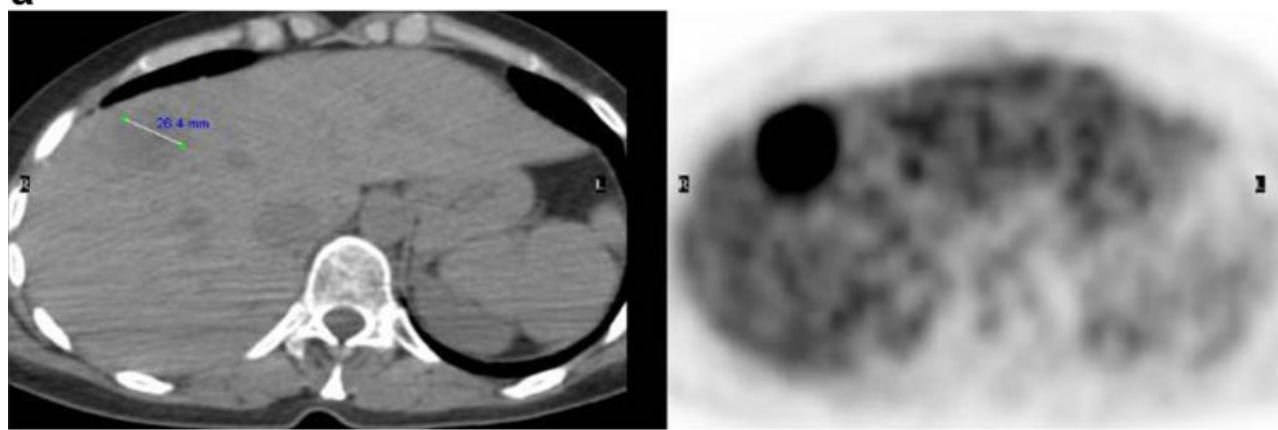

b

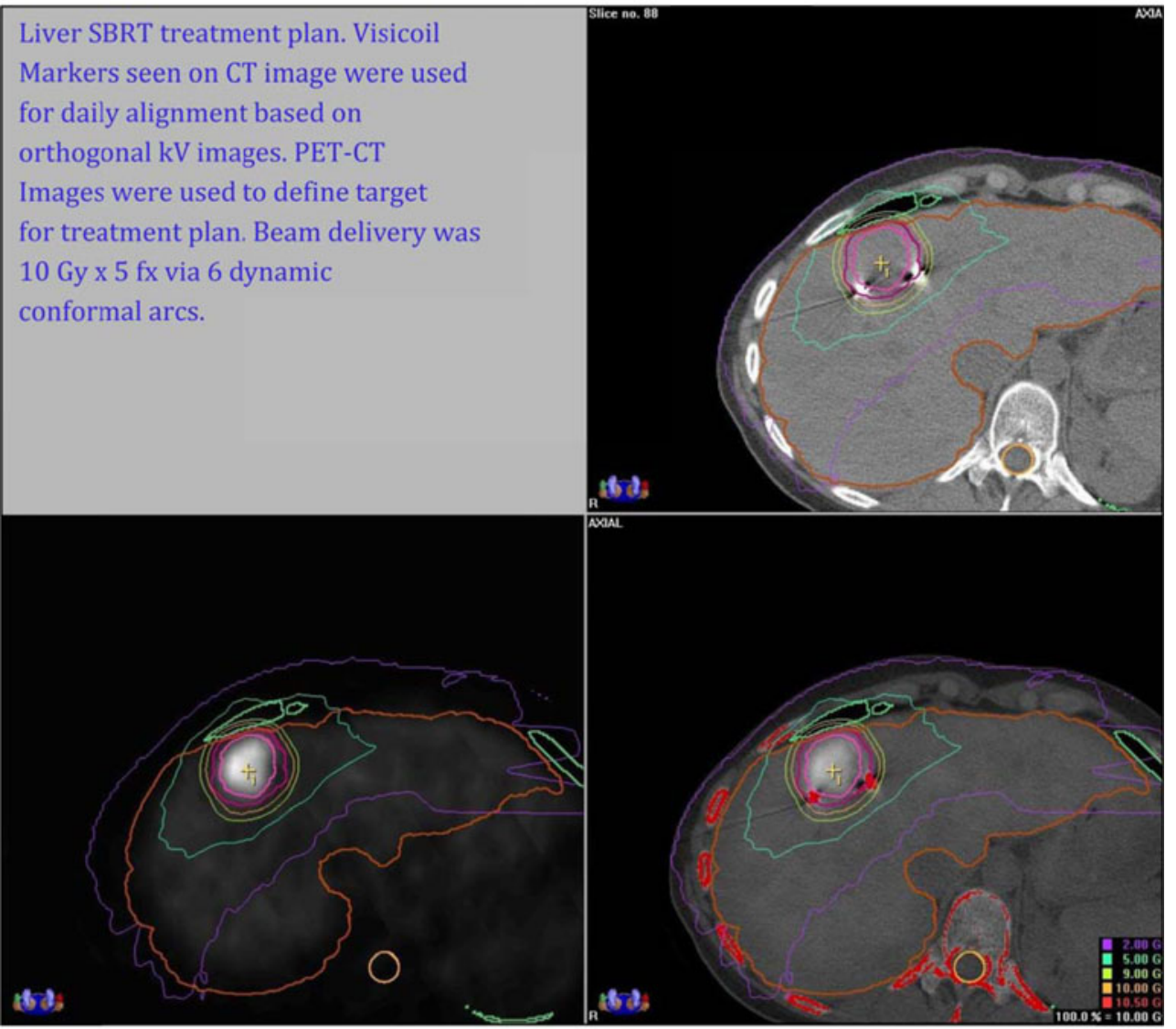

C

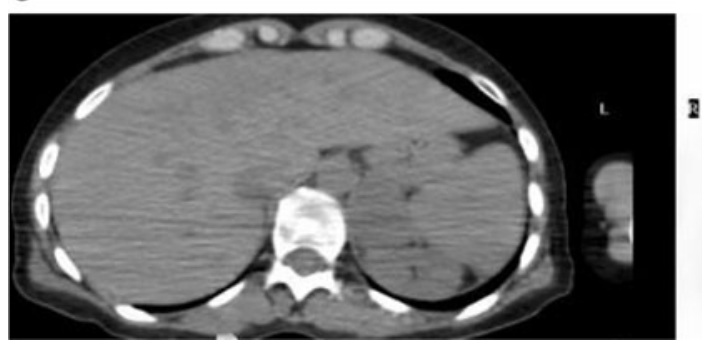


When feasible (i.e., in the absence of immediately adjacent critical structures and in the setting of adequate hepatic reserve) and as illustrated by a clinical case example from Fig. 2, high-dose SBRT for liver metastases offers significant promise. Rusthoven et al. [85] reported on a multiinstitutional phase I-II study for liver metastases. The phase I portion of the study achieved dose escalation from 36 to $60 \mathrm{~Gy}$ in three fractions in increments of 6 Gy without doselimiting toxicity, while the phase II dose was 60 Gy in three fractions. Protocol constraints for normal liver specified that $\geq 700 \mathrm{cc}$ should receive $\leq 15 \mathrm{~Gy}$. The authors described excellent actuarial local control rates of $92 \%$ at 2 years, with improved control among lesions $\leq 3 \mathrm{~cm}$. Serious adverse effects were limited to one case of grade 3 soft tissue toxicity. Objectively, it should be noted that the high reported control rates likely owe both to the high tumoricidal doses and limited target volume, as the median maximum tumor diameter was $2.7 \mathrm{~cm}$. Substantially larger tumors were treated in the University of Toronto series [84] where, among 68 patients, a median SBRT dose of 41.8 Gy was delivered in six fractions over 2 weeks using an individualized prescription formalism based on $V_{\text {eff. In }}$ contrast to the Colorado series, this protocol included more advanced lesions, with median tumor volume of $75.2 \mathrm{cc}$. The individualized prescription schemes proved clinically safe, as no dose-limiting toxicity, radiation-induced liver disease, or other grades 3 to 5 toxicity was observed. Recently, University of Texas Southwestern investigators [86] reported their phase I dose escalation study for SBRT in patients with hepatic metastasis in a prospective institutional trial allowing up to five lesions. The initial protocol delivered $30 \mathrm{~Gy}$ in three fractions with dose escalation to 50 and 60 Gy in five fractions. Hepatic dose-volume constraints required preservation of $\geq 700 \mathrm{cc}$ of normal liver to $\leq 15$ Gy in three fractions, with the threshold tolerance dose modified to $21 \mathrm{~Gy}$ for the five-fraction scheme. Tumor median lesion diameter was $2.5 \mathrm{~cm}$, with median PTV volume of 43 cc. Results demonstrated a significant dose-response between the 30 and 60 Gy cohorts. Local control was $100 \%, 89 \%$, and $56 \%$ at 24 months for the 60,50 , and 30 Gy cohorts, respectively.

Among patients with colorectal carcinoma metastases, initial SBRT reports indicate potentially lower local control rates, presumably attributed to radioresistance. As an example, 3year local control of $74 \%$ was reported by van der Pool et al. [87] among a cohort of patients with median tumor size of $2.3 \mathrm{~cm}$. A multi-institutional pooled analysis by Cheng et al. [88] sought to further explore SBRT outcomes for colorectal liver metastases. Among 65 patients with 102 CRC lesions, $29 \%$ of patients had local infield recurrences, and $68 \%$ of patients exhibited progression outside the liver. Age, BED, dose per fraction, total dose, and maximal lesion size were all identified as significant predictors of local control. Analysis demonstrated that, for a three-fraction SBRT regimen, an estimated 46 to 52 Gy would be required to achieve $90 \%$ local control. In agreement with other series, the pooled analysis demonstrated acceptable toxicity rates inclusive of gastritis, small bowel ulcers, and elevated liver enzymes and persistent chest wall pain.

\section{Conclusions}

Phase III studies have demonstrated substantial benefit for aggressive local and systemic management of primary and secondary liver tumors. Surgery and OLT constitute the gold-standard local therapies for resectable patients in good performance status, although these comprise a relatively small fraction of the population. Among alternative treatments, RT holds promise for treatment of potentially curable nonsurgical candidates and for aggressive palliation of oligometastatic patients. Promising liver RT outcomes utilizing both conventionally fractionated and hypofractionated (SBRT) approaches have been reported and require significant commitment in terms of high-quality imaging and target delineation, treatment planning with attention to normal tissue toxicity, and effective treatment delivery using appropriate immobilization techniques and image-guided delivery. Phase III data are needed to firmly establish the role of RT, particularly in light of alternative and often competing treatment modalities including RFA, chemoembolization, radioembolization, and systemic agents.

Conflict of interest The authors state that there is no conflict of interest.

\section{References}

1. Jemal A et al (2010) Cancer statistics, 2010. CA Cancer J Clin 60 (5):277-300

2. Bosch FX et al (2005) Epidemiology of hepatocellular carcinoma. Clin Liver Dis 9(2):191-211, v

3. Forner A, Reig ME, de Lope CR (2010) Current strategy for staging and treatment: the BCLC update and future prospects. Semin Liver Dis 30(1):61-74

4. Child CT, Turcotte JG (1964) Surgery and portal hypertension. In: The liver and portal hypertension. Philadelphia: Saunders

5. Takayama $\mathrm{T}$ et al (1998) Early hepatocellular carcinoma as an entity with a high rate of surgical cure. Hepatology 28(5):1241-1246

6. Mazzaferro V et al (1996) Liver transplantation for the treatment of small hepatocellular carcinomas in patients with cirrhosis. N Engl J Med 334(11):693-699

7. Park Y-K et al (2009) Hepatic resection for hepatocellular carcinoma meeting Milan criteria in Child-Turcotte-Pugh class a patients with cirrhosis. Transplant Proc 41(5):1691-1697 
8. Sala $\mathrm{M}$ et al (2004) Initial response to percutaneous ablation predicts survival in patients with hepatocellular carcinoma. Hepatology 40(6):1352-1360

9. Llovet JM, Bruix J (2003) Systematic review of randomized trials for unresectable hepatocellular carcinoma: chemoembolization improves survival. Hepatology 37(2):429-442

10. Llovet JM et al (2008) Sorafenib in advanced hepatocellular carcinoma. N Engl J Med 359(4):378-390

11. Cheng A-L et al (2009) Efficacy and safety of sorafenib in patients in the Asia-Pacific region with advanced hepatocellular carcinoma: a phase III randomised, double-blind, placebo-controlled trial. Lancet Oncol 10(1):25-34

12. Weiss L et al (1986) Haematogenous metastatic patterns in colonic carcinoma: an analysis of 1541 necropsies. J Pathol 150(3):195-203

13. Weichselbaum RR, Hellman S (2011) Oligometastases revisited. Nat Rev Clin Oncol 8(6):378-382

14. Saltz LB et al (2000) Irinotecan plus fluorouracil and leucovorin for metastatic colorectal cancer. Irinotecan study group. N Engl J Med 343(13):905-914

15. Scheele J et al (1996) Surgical resection of colorectal liver metastases: gold standard for solitary and radically resectable lesions. Swiss Surg Suppl 4:4-17

16. House MG et al (2010) Survival after hepatic resection for metastatic colorectal cancer: trends in outcomes for 1,600 patients during two decades at a single institution. J Am Coll Surg 210(5):744-752, 752-5

17. Fong Y et al (1999) Clinical score for predicting recurrence after hepatic resection for metastatic colorectal cancer: analysis of 1001 consecutive cases. Ann Surg 230(3):309-318, discussion 318-21

18. Simmonds PC et al (2006) Surgical resection of hepatic metastases from colorectal cancer: a systematic review of published studies. Br J Cancer 94(7):982-999

19. Elias D, Di Pietroantonio D (2006) Surgery for liver metastases from breast cancer. HPB (Oxford) 8(2):97-99

20. Dawson LA (2011) Overview: where does radiation therapy fit in the spectrum of liver cancer local-regional therapies? Semin Radiat Oncol 21(4):241-246

21. Couinaud C (1992) The anatomy of the liver. Ann Ital Chir 63 (6):693-697

22. Voroney J-P et al (2006) Prospective comparison of computed tomography and magnetic resonance imaging for liver cancer delineation using deformable image registration. Int $\mathrm{J}$ Radiat Oncol Biol Phys 66(3):780-791

23. Reed GB Jr, Cox AJ Jr (1966) The human liver after radiation injury. A form of veno-occlusive disease. Am J Pathol 48(4):597-611

24. Fajardo LF, Colby TV (1980) Pathogenesis of veno-occlusive liver disease after radiation. Arch Pathol Lab Med 104(11):584-588

25. Lawrence TS et al (1995) Hepatic toxicity resulting from cancer treatment. Int J Radiat Oncol Biol Phys 31(5):1237-1248

26. Guha C, Kavanagh BD (2011) Hepatic radiation toxicity: avoidance and amelioration. Semin Radiat Oncol 21(4):256-263

27. You CR, Jang JW, Choi JK et al (2010) Hepatic failure caused by reactivation of YMDD mutants occurring during preemptive lamivudine therapy. Gut Liver 4(2):262-265

28. Richardson PG et al (2010) Defibrotide for the treatment of severe hepatic veno-occlusive disease and multiorgan failure after stem cell transplantation: a multicenter, randomized, dose-finding trial. Biol Blood Marrow Transplant 16(7):1005-1017

29. Corbacioglu S et al. (2009) Defibrotide (DF) for the prevention of hepatic veno-occlusive disease (VOD) in pediatric stem cell transplantion: results of a prospective phase II/III randomized, multicenter study. ASH; Abstract 653

30. Borgelt BB et al (1981) The palliation of hepatic metastases: results of the radiation therapy oncology group pilot study. Int $\mathrm{J}$ Radiat Oncol Biol Phys 7(5):587-591

31. Russell AH et al (1993) Accelerated hyperfractionated hepatic irradiation in the management of patients with liver metastases: results of the RTOG dose escalating protocol. Int J Radiat Oncol Biol Phys 27(1):117-123

32. Emami B et al (1991) Tolerance of normal tissue to therapeutic irradiation. Int J Radiat Oncol Biol Phys 21(1):109-122

33. Robertson JM et al (1997) A phase I trial of hepatic arterial bromodeoxyuridine and conformal radiation therapy for patients with primary hepatobiliary cancers or colorectal liver metastases. Int J Radiat Oncol Biol Phys 39(5):1087-1092

34. Burman C et al (1991) Fitting of normal tissue tolerance data to an analytic function. Int J Radiat Oncol Biol Phys 21(1):123-135

35. Lawrence TS et al (1992) The use of 3-D dose volume analysis to predict radiation hepatitis. Int J Radiat Oncol Biol Phys 23(4):781-788

36. Dawson LA et al (2002) Analysis of radiation-induced liver disease using the Lyman NTCP model. Int J Radiat Oncol Biol Phys 53(4):810-821

37. Dawson LA, Ten Haken, Randall K (2005) Partial volume tolerance of the liver to radiation. Semin Radiat Oncol 15(4):279-283

38. Ten Haken RK, Lawrence TS, Dawson LA (2006) Prediction of radiation-induced liver disease by Lyman normal-tissue complication probability model in three-dimensional conformal radiation therapy for primary liver carcinoma: in regards to $\mathrm{Xu}$ et al. (Int J Radiat Oncol Biol Phys 2006;65:189-195). Int J Radiat Oncol Biol Phys 66(4):1272, author reply 1272-1272; author reply 1273

39. Cheng JC, Wu JK, Huang CM et al (2002) Radiation-induced liver disease after radiotherapy for hepatocellular carcinoma: clinical manifestation and dosimetric description. Radiother Oncol 63(1):41-50

40. Cheng JC-H et al (2004) Biologic susceptibility of hepatocellular carcinoma patients treated with radiotherapy to radiation-induced liver disease. Int J Radiat Oncol Biol Phys 60(5):1502-1509

41. Chou $\mathrm{CH}$ et al (2007) Radiation-induced hepatitis B virus reactivation in liver mediated by the bystander effect from irradiated endothelial cells. Clin Cancer Res 13(3):851-857

42. Koo JE et al (2010) Combination of transarterial chemoembolization and three-dimensional conformal radiotherapy for hepatocellular carcinoma with inferior vena cava tumor thrombus. Int $\mathbf{J}$ Radiat Oncol Biol Phys 78(1):180-187

43. Huang Y-J et al (2009) The treatment responses in cases of radiation therapy to portal vein thrombosis in advanced hepatocellular carcinoma. Int J Radiat Oncol Biol Phys 73(4):1155-1163

44. Hamad A, Aziz A et al (2009) Stereotactic radiotherapy of the liver: a bridge to transplantation stereotactic radiotherapy of the liver: a bridge to transplantation. Technol Cancer Res Treat 8(6):401-405

45. Sandroussi $C$ et al (2010) Radiotherapy as a bridge to liver transplantation for hepatocellular carcinoma. Transpl Int 23(3):299-306

46. Kim TH et al (2006) Three-dimensional conformal radiotherapy of unresectable hepatocellular carcinoma patients for whom transcatheter arterial chemoembolization was ineffective or unsuitable. Am J Clin Oncol 29(6):568-575

47. Guo W-J et al (2003) Comparison between chemoembolization combined with radiotherapy and chemoembolization alone for large hepatocellular carcinoma. World J Gastroenterol 9(8):1697-1701

48. Meng M-B et al (2009) Transcatheter arterial chemoembolization in combination with radiotherapy for unresectable hepatocellular carcinoma: a systematic review and meta-analysis. Radiother Oncol 92(2):184-194

49. Mornex F et al (2006) Feasibility and efficacy of high-dose threedimensional-conformal radiotherapy in cirrhotic patients with small-size hepatocellular carcinoma non-eligible for curative therapies - mature results of the French phase II RTF-1 trial. Int J Radiat Oncol Biol Phys 66(4):1152-1158

50. Mornex $\mathrm{F}$ et al (2005) Tolerance and efficacy of conformal radiotherapy for hepatocellular carcinoma in cirrhotic patients. Results of the French RTF1 phase II trial. Cancer Radiother 9(6-7):470-476

51. Hata $\mathrm{M}$ et al (2006) Proton beam therapy for hepatocellular carcinoma patients with severe cirrhosis. Strahlenther Onkol 182 (12):713-720 
52. Girard N, Mornex F (2011) Sorafenib and radiotherapy association for hepatocellular carcinoma. Cancer Radiother 15(1):77-80

53. Chung $C$ et al (2010) Radiation recall dermatitis triggered by multi-targeted tyrosine kinase inhibitors: sunitinib and sorafenib. Anticancer Drugs 21(2):206-209

54. Potters L et al (2010) American Society for Therapeutic Radiology and Oncology (ASTRO) and American College of Radiology (ACR) practice guideline for the performance of stereotactic body radiation therapy. Int J Radiat Oncol Biol Phys 76(2):326-332

55. Brock KK (2011) Imaging and image-guided radiation therapy in liver cancer. Semin Radiat Oncol 21(4):247-255

56. Beddar AS et al (2008) 4D-CT imaging with synchronized intravenous contrast injection to improve delineation of liver tumors for treatment planning. Radiother Oncol 87(3):445-448

57. Wang $\mathrm{W}$ et al (2010) Prospective evaluation of microscopic extension using whole-mount preparation in patients with hepatocellular carcinoma: definition of clinical target volume for radiotherapy. Radiat Oncol 5:73

58. Bi A-H et al (2010) Impact factors for microinvasion in intrahepatic cholangiocarcinoma: a possible system for defining clinical target volume. Int J Radiat Oncol Biol Phys 78(5):1427-1436

59. Mendez Romero A et al (2012) Comparison of macroscopic pathology measurements with magnetic resonance imaging and assessment of microscopic pathology extension for colorectal liver metastases. Int J Radiat Oncol Biol Phys 82(1):159-166

60. Keall PJ et al (2006) The management of respiratory motion in radiation oncology report of AAPM task group 76. Med Phys 33 (10):3874-3900

61. Balter JM et al (1998) Improvement of CT-based treatmentplanning models of abdominal targets using static exhale imaging. Int J Radiat Oncol Biol Phys 41(4):939-943

62. Eccles CL et al (2011) Interfraction liver shape variability and impact on GTV position during liver stereotactic radiotherapy using abdominal compression. Int J Radiat Oncol Biol Phys 80(3):938-946

63. Case RB et al (2010) Interfraction and intrafraction changes in amplitude of breathing motion in stereotactic liver radiotherapy. Int J Radiat Oncol Biol Phys 77(3):918-925

64. Case RB et al (2009) Inter- and intrafraction variability in liver position in non-breath-hold stereotactic body radiotherapy. Int $\mathrm{J}$ Radiat Oncol Biol Phys 75(1):302-308

65. Eccles $\mathrm{C}$ et al (2006) Reproducibility of liver position using active breathing coordinator for liver cancer radiotherapy. Int J Radiat Oncol Biol Phys 64(3):751-759

66. Hawkins MA et al (2006) Assessment of residual error in liver position using $\mathrm{kV}$ cone-beam computed tomography for liver cancer high-precision radiation therapy. Int $\mathrm{J}$ Radiat Oncol Biol Phys 66(2):610-619

67. Kothary N et al (2009) Safety and efficacy of percutaneous fiducial marker implantation for image-guided radiation therapy. J Vasc Interv Radiol 20(2):235-239

68. Hennessey H et al (2009) Cardiac embolization of an implanted fiducial marker for hepatic stereotactic body radiotherapy: a case report. J Med Case Reports 3:140

69. Sonke J-J et al (2005) Respiratory correlated cone beam CT. Med Phys 32(4):1176-1186

70. Park HC et al (2002) Dose-response relationship in local radiotherapy for hepatocellular carcinoma. Int J Radiat Oncol Biol Phys 54(1):150-155
71. Park W et al (2005) Local radiotherapy for patients with unresectable hepatocellular carcinoma. Int J Radiat Oncol Biol Phys 61 (4): $1143-1150$

72. Park SH et al (2005) Salvage chemotherapy with irinotecan and cisplatin in patients with metastatic gastric cancer failing both 5fluorouracil and taxanes. Anticancer Drugs 16(6):621-625

73. Blomgren $\mathrm{H}$ et al (1995) Stereotactic high dose fraction radiation therapy of extracranial tumors using an accelerator. Clinical experience of the first thirty-one patients. Acta Oncol 34(6):861-870

74. Herfarth KK et al (2001) Stereotactic single-dose radiation therapy of liver tumors: results of a phase I/II trial. J Clin Oncol 19(1):164-170

75. Wulf J et al (2006) Stereotactic radiotherapy of primary liver cancer and hepatic metastases. Acta Oncol 45(7):838-847

76. Tse RV et al (2008) Phase I study of individualized stereotactic body radiotherapy for hepatocellular carcinoma and intrahepatic cholangiocarcinoma. J Clin Oncol 26(4):657-664

77. Goodman KA et al (2010) Dose-escalation study of single-fraction stereotactic body radiotherapy for liver malignancies. Int J Radiat Oncol Biol Phys 78(2):486-493

78. Goyal K et al (2010) Cyberknife stereotactic body radiation therapy for nonresectable tumors of the liver: preliminary results. HPB Surg, 2010. doi:10.1155/2010/309780

79. Kwon JH et al (2010) Long-term effect of stereotactic body radiation therapy for primary hepatocellular carcinoma ineligible for local ablation therapy or surgical resection. Stereotactic radiotherapy for liver cancer. BMC Cancer 10:475

80. Son SH et al (2010) Stereotactic body radiotherapy for patients with unresectable primary hepatocellular carcinoma: dose-volumetric parameters predicting the hepatic complication. Int J Radiat Oncol Biol Phys 78(4):1073-1080

81. Cárdenes HR et al (2010) Phase I feasibility trial of stereotactic body radiation therapy for primary hepatocellular carcinoma. Clin Transl Oncol 12(3):218-225

82. Facciuto M, Singh MK et al (2012) Stereotactic body radiation therapy in hepatocellular carcinoma and cirrhosis: evaluation of radiological and pathological response. J Surg Oncol 105(7):692-698

83. Hoyer M et al (2006) Phase II study on stereotactic body radiotherapy of colorectal metastases. Acta Oncol 45(7):823-830

84. Rusthoven KE et al (2009) Multi-institutional phase I/II trial of stereotactic body radiation therapy for liver metastases. J Clin Oncol 27(10):1572-1578

85. Lee MT et al (2009) Phase I study of individualized stereotactic body radiotherapy of liver metastases. J Clin Oncol 27(10):1585-1591

86. Rule W et al (2011) Phase I dose-escalation study of stereotactic body radiotherapy in patients with hepatic metastases. Ann Surg Oncol 18(4):1081-1087

87. van der Pool AEM et al (2010) Stereotactic body radiation therapy for colorectal liver metastases. Br J Surg 97(3):377-382

88. Chang DT et al (2011) Stereotactic body radiotherapy for colorectal liver metastases: a pooled analysis. Cancer 117 (17):4060-4069

89. Sherman DM et al (1978) Palliation of hepatic metastasis. Cancer 41(5):2013-2017

90. Mohiuddin M, Chen E, Ahmad N (1996) Combined liver radiation and chemotherapy for palliation of hepatic metastases from colorectal cancer. J Clin Oncol 14(3):722-728 